\title{
Une analyse diachronique des pratiques funéraires préhistoriques du centre du Vanuatu
}

Frédérique Valentin, Matthew Spriggs, Stuart Bedford et Hallie Buckley

\section{Q OpenEdition \\ 12 Journals}

Édition électronique

URL : http://journals.openedition.org/jso/5773

DOI : $10.4000 /$ jso. 5773

ISSN : $1760-7256$

Éditeur

Société des océanistes

Édition imprimée

Date de publication : 30 juin 2009

Pagination : 39-52

ISBN : 978-2-85430-024-6

ISSN : 0300-953x

Référence électronique

Frédérique Valentin, Matthew Spriggs, Stuart Bedford et Hallie Buckley, « Une analyse diachronique des pratiques funéraires préhistoriques du centre du Vanuatu », Journal de la Société des Océanistes [En ligne], 128 | janvier-juin 2009, mis en ligne le 30 juin 2012, consulté le 21 avril 2019. URL : http:// journals.openedition.org/jso/5773; DOI : 10.4000/jso.5773

(c) Tous droits réservés 


\title{
Une analyse diachronique des pratiques funéraires préhistoriques du centre du Vanuatu
}

\author{
par \\ Frédérique VALENTIN*, Matthew SPRIGGS**, Stuart BEDFORD** \\ et Hallie BUCKLEY***
}

\section{RÉSUMÉ}

Les travaux de José Garanger dans le centre du Vanuatu (1963-1967) permirent la découverte et l'étude archéologique de nombreuses sépultures sur les îles d'Efate et de Tongoa, contribuant à étendre les connaissances sur les pratiques funéraires dans l'archipel au deuxième millénaire après J.-C. La découverte, à Teouma sur l'île d'Efate en 2004, d'un cimetière Lapita ancien ajoute des informations sur les pratiques les plus anciennes. L'ensemble sépulcral de Teouma, actuellement connu par une cinquantaine de structures funéraires, se caractérise par des orientations et des positions de dépôt très variées et un traitement uniforme mais complexe du corps et des os. S'appuyant principalement sur ces deux ensembles de données, l'article ébauche une analyse diachronique des pratiques funéraires préhistoriques du Vanuatu basée sur cinq composantes particulières du dispositif funéraire dont José Garanger avait remarqué l'importance : traitement du corps et des ossements, position initiale du défunt, port de parure et association de mobilier, usage de sépulture multiple et orientation de la sépulture.

MoTS-CLÉS : sépulture, squelette humain, Vanuatu, chronologie préhistorique, Teouma, Lapita, deuxième millénaire après J.-C.

Les travaux menés par José Garanger, entre 1963 et 1967, sur les îles d'Efate et de Tongoa

\section{ABSTRACT}

The research conducted by José Garanger in central Vanuatu (1963-1967), led to the discovery and archaeological study of a number of burials on the islands of Efate and Tongoa, and contributed to increased knowledge of burial practices of second millennium AD communities of the archipelago. The discovery in 2004 of the early Lapita cemetery at Teouma, on Efate Island, supplements this knowledge with a new data set. The cemetery, currently known by about fifty funerary features, is characterized by diverse body orientations and positions, and homogenous but complex body and bone treatments. Relying primarily on these two sets of data, this paper outlines a diachronic analysis of the Vanuatu prehistoric burial practices using five attributes of the funerary system that were assessed as significant by José Garanger: body and bone treatment, original position of the deceased, ornaments and associated artefacts, use of multiple burial and burial orientation.

KeYwORDs: burial practices, human skeleton, Vanuatu, prehistoric sequence, Teouma, Lapita, second millennium $\mathrm{AD}$

dans le centre du Vanuatu, permirent la mise au jour et l'étude archéologique d'une centaine de

\footnotetext{
* CNRS UMR 7041, Nanterre, France, frederique.valentin@mae.u-paris10.fr.

** The Australian National University, Canberra, Australie.

*** University of Otago, Dunedin, Nouvelle-Zélande.
} 
sépultures attribuables au deuxième millénaire après J.-C. (Garanger, 1972). La découverte en 2004 dans la même région, sur l'île d'Efate (figure 1), du cimetière Lapita de Teouma, daté d'environ 1050 ans avant J.-C. (Bedford et al., 2004, 2006, sous presse), incite à ébaucher une mise en perspective diachronique de données funéraires.

Les gestes, les pratiques et les moyens mis en œuvre pour disposer du défunt et de ses restes se sont-ils modifiés au cours des trois mille ans que recouvre la chronologie préhistorique de l'archipel ? Le changement de la culture matérielle visible autour de 1000 ans après J.-C. dans le centre du Vanuatu (Garanger, 1972; Spriggs, 1997 ; Bedford, 2006) trouve-t-il un écho dans les pratiques funéraires? Les influences polynésiennes, s'exprimant à travers les parures portées par les défunts d'Efate (Garanger, 1972) mais aussi d'Aneityum (Spriggs, 1997) et de Futuna (Shutler et al., 2002) dans le sud de l'archipel, ont-elles aussi marqué les pratiques et les modes de traitement du défunt? Prenant appui sur les résultats publiés par José Garanger (1972) ainsi que sur ceux obtenus par Graeme Ward et Philip Houghton (1988, 1991), Matthew Spriggs (1997) et Richard Shutler et al. (2002), le présent article propose une analyse reposant sur cinq composantes du système funéraire dont José Garanger avait remarqué l'importance. Il s'agit du traitement du corps et des ossements, de la position initiale du défunt, du port de parure et de l'association de mobilier, de l'usage de sépulture multiple et de l'orientation de la sépulture.

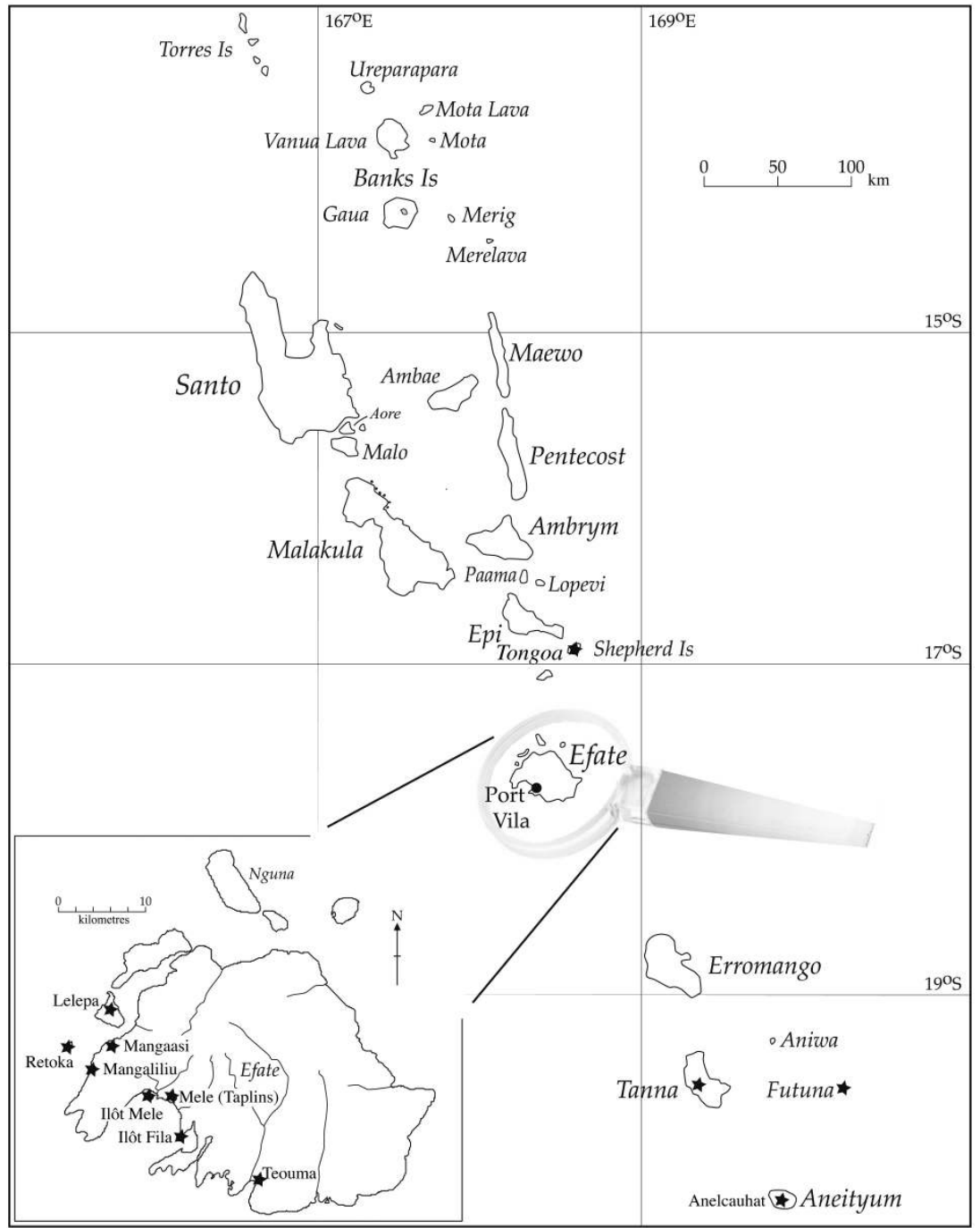

FIGURE 1. - Carte localisant les sites funéraires mentionnés dans cette étude 


\section{Sépultures du deuxième millénaire après J.-C.}

Les sépultures décrites par José Garanger (1972) ont été mises au jour dans les régions d'Efate et de Tongoa où quinze sites funéraires et/ou sacrés ont été fouillés (tableau 1, figure 1). Évaluer leur nombre est compliqué par la complexité des sépultures multiples découvertes. Toutefois, 118 dépôts sépulcraux peuvent être individualisés. Ils livrèrent les restes de 15 en- fants, principalement morts en bas-âge, et de 56 adultes dont 24 hommes et 26 femmes (le sexe des autres individus n'étant pas précisé). Ces sépultures se rapportent toutes au deuxième millénaire après $\mathrm{J}$-C. et, plus probablement à la seconde moitié de celui-ci. Les inhumations de Retoka, précédemment attribuées au XIII ${ }^{\mathrm{e}}$ siècle de l'ère chrétienne (Garanger, 1972), sont maintenant datées du XVII ${ }^{\mathrm{e}}$ (Bedford et al., 1998 ; Bedford, 2006).

\begin{tabular}{|c|c|c|c|c|}
\hline Île & Site & Nombre de sépulture & Nombre de corps & Âge au décès et sexe \\
\hline Lelepa & $\begin{array}{ll}\text { Grotte de } \\
\text { Mortutu }\end{array}$ & 1 & 1 & 1 adulte de sexe indéterminé \\
\hline Lelepa & Wele & 1 & 1 & 1 adulte de sexe indéterminé \\
\hline Lelepa & EF 101-5 & 1 (double) & 2 & 1 enfant, 1 femme \\
\hline Lelepa & EF 101-6 & 2 & 2 & 2 adultes de sexe indéterminé \\
\hline Lelepa & Taupele & 1 & 1 & 1 adulte ? de sexe indéterminé \\
\hline Lelepa & Smouta & 2 & 2 & 2 adultes ? de sexe indéterminé \\
\hline Retoka & Niveau I & 3 & 3 (2 sont secondaires) & 3 enfants en bas âge \\
\hline Retoka & Niveau II & 1 multiple & 40 ( 3 sont secondaires) & $\begin{array}{l}1 \text { enfant, } 16 \text { hommes, } 15 \text { femmes, } 8 \text { adultes } \\
\text { de sexe indéterminé }\end{array}$ \\
\hline Retoka & Niveau III & 1 multiple & $\begin{array}{l}6 \text { ( } 1 \text { est secondaire, } \\
\text { contenant aussi } 1 \text { cochon })\end{array}$ & $\begin{array}{l}3 \text { hommes, } 2 \text { femmes, } 1 \text { adulte de sexe } \\
\text { indéterminé }\end{array}$ \\
\hline Efate & Mangaasi & 5 & 5 & 5 adultes ? de sexe indéterminé \\
\hline Ilot Mele & $\begin{array}{l}\text { Fare Tabu, } \\
\text { Fare Vaitere et } \\
\text { zone sud de } \\
\text { l'îlot }\end{array}$ & 25 (dont 1 double) & $\begin{array}{l}26 \text { (1 double contenant } \\
\text { aussi } 1 \text { oiseau })\end{array}$ & $\begin{array}{l}10 \text { enfants, } 3 \text { femmes, } 13 \text { adultes de sexe } \\
\text { indéterminé }\end{array}$ \\
\hline Tongoa & $\begin{array}{l}\text { Mangarisu To } \\
28-3\end{array}$ & 7 & 8 ( 1 est secondaire) & 8 adultes? de sexe indéterminé \\
\hline Tongoa & $\begin{array}{l}\text { Mangarisu To } \\
29-1(33)\end{array}$ & 1 multiple & 5 & $\begin{array}{l}1 \text { homme, } 3 \text { femmes, } 1 \text { adulte de sexe } \\
\text { indéterminé }\end{array}$ \\
\hline Tongoa & $\begin{array}{l}\text { Mangarisu To } \\
29-1 \\
2,5,7\end{array}$ & 7 & 7 & $\begin{array}{l}2 \text { hommes, } 2 \text { adultes de sexe indéterminé, } \\
3 \text { adultes? de sexe indéterminé }\end{array}$ \\
\hline Tongoa & Panita To 51 & 6 & 6 & 6 adultes de sexe indéterminé \\
\hline Tongoa & $\begin{array}{l}\text { Panita Ti Ton- } \\
\text { goa } \\
\text { Liseiriki }\end{array}$ & 1 multiple? & 6 & $\begin{array}{l}2 \text { hommes, } 2 \text { femmes, } 2 \text { adultes de sexe } \\
\text { indéterminé }\end{array}$ \\
\hline Total & 15 sites & $\begin{array}{l}59 \text { sépultures } \\
\text { individuelles } \\
2 \text { sépultures doubles } \\
3 \text { ou } 4 \text { sépultures } \\
\text { multiples }\end{array}$ & $\begin{array}{l}118 \text { inhumations } \\
\text { humaines } \\
2 \text { inhumations animales } \\
\text { ( } 1 \text { cochon, } 1 \text { oiseau) }\end{array}$ & $\begin{array}{l}15 \text { enfants } \\
24 \text { hommes } \\
26 \text { femmes } \\
37 \text { adultes de sexe indéterminé } \\
19 \text { adultes ? de sexe indéterminé }\end{array}$ \\
\hline
\end{tabular}

TABLEAU 1. - Inventaire de sites funéraires fouillés par José Garanger (1972), nombre de sépultures, de défunts, sexe et âge au décès

\section{Traitement du corps}

L'inhumation est le mode de traitement choisi pour faire disparaître le corps du défunt (Thomas, 1980). La pratique semble régulière dans la région Efate-Shepherd durant le second millénaire après J.-C. José Garanger (1972) ne signale pas d'incinération, même s'il a découvert des ossements brûlés et fragmentaires dans le site de Mangaasi.

\section{Position initiale du corps}

Les positions d'inhumation se caractérisent par une faible diversité et des récurrences (tableau 2). Le défunt a été déposé sur le dos dans $56 \%$ des cas, les membres supérieurs étendus le long du corps, les membres inférieurs allongés ou légèrement fléchis, parallèles l'un à l'autre. L'attitude paraît plus constante dans les sites constitués de sépultures individuelles ou 
doubles des îlots Lelepa et Mele et dans le site To 28-3 de Tongoa. La flexion des membres, au moins en partie corrélée à la position du corps, traduit un traitement différent des hommes et des femmes. Elle est aussi liée à la multiplicité des dépôts dans la tombe. Une forte flexion des membres supérieurs apparaît plus fréquemment dans les sites de Retoka et To 29-1 (33) de Mangarisu où les corps (sur le côté et sur le ventre) ont été déposés simultanément, selon une scénographie hiérarchisée autour d'un personnage central.

\begin{tabular}{|c|c|c|}
\hline Positions & Fréquence & Aspects particuliers \\
\hline Sur le dos & $94 / 107$ & \\
\hline Sur le côté & $11 / 107$ & $\begin{array}{l}\text { sur le côté droit ou gauche, } \\
9 \text { cas à Retoka, } 2 \text { cas sur le site To 29-1 (33) de Mangarisu }\end{array}$ \\
\hline Sur le ventre & $2 / 107$ & 2 femmes, l'une à Retoka et l'autre sur le site To 29-1 (33) de Mangarisu \\
\hline Position fœtale & 2 & 2 nouveau-nés, l'un à Retoka, l'autre au Fare Vaitere (îlot Mele) \\
\hline $\begin{array}{l}\text { Membres supérieurs } \\
\text { étendus le long du corps }\end{array}$ & $76 / 100$ & \\
\hline $\begin{array}{l}\text { Membres supérieurs } \\
\text { flexion du coude }\end{array}$ & $21 / 100$ & $\begin{array}{l}\text { * flexion plus ou moins marquée ramenant la main sur la poitrine, } 3 \text { cas à l'îlot } \\
\text { Mele, } 2 \text { à l'ilot Lelepa, } 5 \text { à Retoka, } 1 \text { sur le site To 29-1 (33) de Mangarisu et } 1 \text { dans } \\
\text { sépulture de Ti Tongoa Liseiriki } \\
\text { * hyperflexion ramenant une main, ou les deux, devant le visage ou sur l'épaule, } \\
6 \text { cas à Retoka, } 3 \text { cas sur le site To 29-1 (33) de Mangarisu }\end{array}$ \\
\hline $\begin{array}{l}\text { Membres supérieurs } \\
\text { en abduction }\end{array}$ & $3 / 100$ & 3 cas à Retoka \\
\hline $\begin{array}{l}\text { Membres inférieurs } \\
\text { allongés }\end{array}$ & $69 / 100$ & \\
\hline $\begin{array}{l}\text { Membres inférieurs : } \\
\text { flexion de la hanche et du genou }\end{array}$ & $29 / 100$ & $\begin{array}{l}\text { sur le côté ou verticalement, degré de flexion varié, souvent faible, parfois modéré } \\
\text { et n'atteignant jamais l'hyperflexion }\end{array}$ \\
\hline
\end{tabular}

TABLEAU 2. - Inventaire et fréquence des positions d'inhumation observées dans les sépultures fouillées par José Garanger (1972)

\section{Parure et mobilier}

Décrits et illustrés en détail par José Garanger (1972), les ornements utilisés pour parer les défunts sont très divers, très fréquents voire constants dans les sépultures de la côte nordouest d'Efate où ils apparaissent en quantité variable, d'un à une dizaine voire plus par défunt. À l'opposé, les ornements sont peu variés, rarement présents et peu abondants dans les sépultures de Tongoa. Ainsi, l'usage de bracelet en dent de cochon semble récurrent à Tongoa alors qu'il n'apparaît que dans deux sites d'Efate (Retoka et Taupele à Lelepa). Comme l'avait déjà souligné José Garanger (1972 : 115), ces objets de parure sont absents dans les sites céramiques plus anciens et certains d'entre eux pourraient indiquer une influence polynésienne.

Les éléments mobiliers sont rares, tant dans les sépultures d'Efate que dans celles de Tongoa. Ils comprennent une grande herminette en Tridacna et des nacres ouvragées (Retoka), des pierres magiques façonnées dans du corail ou du basalte (Retoka, Mangarisu) ainsi que divers coquillages non modifiés : lucines, arches, tellines ou turbo, identifiés à Retoka, Mangaasi et Mangarisu. À cet inventaire d'objets s'ajoute le dépôt primaire du corps d'un animal dans cer- taines sépultures: un cochon à Retoka, un oiseau sur l'îlot Mele.

\section{Sépultures multiples et morts d'accompagnement}

Le corpus réuni par José Garanger comprend cinq sépultures multiples dont deux sont doubles et trois comptent plus de cinq sujets. D'un point de vue archéologique, la présence de plusieurs défunts déposés simultanément dans une même tombe implique des décès concomitants (si les corps n'ont pas subi de momification) et engage à s'interroger sur la (ou les) cause(s) des morts.

Dans les cas de Retoka et de Mangarisu (Garanger, 1972 : 58-77, 91-92, fig. 145, 152-177, 198, 247, 257), il existe quatre faits remarquables : emplacement de la sépulture dans un lieu particulier : îlot peu accessible pour Retoka, lieu sacré pour TO 29-1 ; mort simultanée d'un nombre important, voire très important, d'adultes répartis équitablement selon les sexes (19 hommes et 17 femmes identifiés à Retoka et 2 hommes et 3 femmes signalés à TO 29-1); agencement des corps selon une mise en scène structurée autour d'un personnage principal en position centrale ; et traitement différent selon le sexe, les hommes reposant sur le dos membres 
étendus et les femmes reposant sur le dos, le côté et le ventre, les membres et le corps fléchis, dans des attitudes non observées dans les autres sépultures du corpus. Ces quatre faits suggèrent un événement spécifique ayant conduit à des morts non naturelles. Ces dernières seraient rituelles si la définition proposée par Albert et al. (2000) était suivie. De plus, les sépultures de Retoka et de Mangarisu présentent une asymétrie funéraire repérable à au moins deux niveaux : celui de la posture qui évoque l'assujettissement des femmes, et celui de l'organisation. L'asymétrie d'organisation est indiquée par le dépôt d'une femme perpendiculairement au personnage principal, au niveau de la tête ou des pieds (niveau III de Retoka et TO 29-1), par la présence d'un dépôt secondaire entre les jambes, près des pieds du personnage principal (niveau III de Retoka) et par la présence de couples aux pieds du personnage principal (Retoka). Cette asymétrie funéraire suggère, selon les termes de Testart (2004), des morts d'accompagnement. Les défunts entourant le personnage central auraient été tués pour l'accompagner dans la mort.

La situation présentée par la sépulture de $\mathrm{Ti}$ Tongoa Liseiriki (Garanger, 1972: 92-94, fig. 261-262) est plus délicate à interpréter. L'ensemble montre, si la simultanéité des dépôts est admise, la combinaison de trois particularités qui pourrait également évoquer des morts d'accompagnement. Ces trois particularités sont: une possible anomalie démographique avec six décès synchrones d'adultes dont ceux de deux hommes et de deux femmes ; un arrangement radiaire des inhumations montrant une tendance à la hiérarchisation dans la disposition relative des défunts (une femme est allongée perpendiculairement aux pieds du personnage sup- posé principal, caractérisé, quant à lui, par une position différente de celle des autres défunts) ; et la présence d'un dépôt secondaire d'ossements sur le thorax.

Les sépultures doubles de Lelepa et de l'îlot Mele associent une femme à un jeune enfant (Garanger, 1972 : 34, 41). Dans le site EF 101-5 de Lelepa, le jeune enfant en position fœtale avait été déposé sur l'avant-bras gauche de la femme reposant sur le dos, les membres étendus. Dans le site de l'îlot Mele, le nouveau-né en position fotale avait été déposé entre les pieds d'une jeune femme, lui faisant face. Cette dernière avait été allongée sur le dos, les avant-bras repliés sur la poitrine et tenant un oiseau dans les mains. La mise en scène et l'asymétrie funéraire observées dans le second cas évoque l'idée d'un accompagnement. Toutefois, il n'est pas possible d'exclure ici l'éventualité de deux morts naturelles simultanées, la mortalité infantile pouvant avoir été élevée à l'époque du contact européen, période à laquelle a eu lieu l'enterrement comme l'indiquent les parures en verroterie portées par les défunts.

\section{Orientation}

La compilation des données relatives aux orientations montre une nette prépondérance de l'axe nord-est/sud-ouest et de la localisation du crâne au nord-est de la sépulture alors que 7 axes possibles et 9 directions possibles pour la tête ont été identifiés (tableau 3). L'axe nord-ouest/sudest est plus rarement observé, le crâne n'étant jamais au sud-est. Une partie de la diversité observée résulte d'une différence entre les îles d'Efate et de Tongoa. Les orientations couramment utilisées à Efate ne le sont pas à Tongoa.

\begin{tabular}{|l|c|c|c|c|c|c|c|c|c|}
\hline Site & NW/SE & W/E & E/W & NEE/SWW & NE/SW & NNE/SSW & S/N & SSW/NNE & SW/NE \\
\hline Mele & & & 4 & 2 & 15 & 3 & & & \\
\hline Lelepa & & 3 & & & 4 & 1 & & & \\
\hline Retoka & 2 & & & 10 & 21 & 11 & & & \\
\hline Mangaasi & & & & & & 5 & & & \\
\hline $\begin{array}{l}\text { Mangarisu } \\
\text { To 28-3 }\end{array}$ & 4 & 3 & & & & & & & \\
\hline $\begin{array}{l}\text { Mangarisu } \\
\text { To 29-1 (33) }\end{array}$ & 1 & 3 & & & & 1 & & & \\
\hline $\begin{array}{l}\text { Mangarisu } \\
\text { To 29-1 }\end{array}$ & 1 & 3 & & & & & & & 3 \\
\hline Panita To 51 & & & 6 & & & & & & \\
\hline $\begin{array}{l}\text { Ti Tongoa } \\
\text { Liseiriki }\end{array}$ & 1 & & & & 1 & & 1 & 3 & \\
\hline Total & 9 & 12 & 10 & 12 & 41 & 21 & 1 & 3 & 3 \\
\hline
\end{tabular}

TABleAu 3. - Orientation des défunts dans les sépultures fouillées par José Garanger (1972) 
D'après José Garanger (1972, 1997), les orientations des sépultures de Mangaasi et des îlots Mele, Lelepa et Retoka, qui apparaissent différentes d'un site à l'autre, convergeraient toutes vers un même point, situé au large du lieu-dit la Pointe du Diable au sud-ouest d'Efate, constituant selon la tradition l'entrée du pays des morts : Bangona (Guiart, 1973). José Garanger observe par ailleurs que les orientations des sépultures de Tongoa sont plus variées, un fait qu'il suppose en relation avec la diversité culturelle actuellement perceptible sur l'île.

L'analyse des orientations des sépultures peut aussi être abordée différemment. En effet, le système d'orientation utilisé dans l'aire océanienne n'est que rarement superposable au système cardinal (Codrington, 1885 : 165). Dans cette aire, il existe un système de structuration de l'espace utilisant des repères terrestres (géocentré) qui, d'après les reconstructions linguistiques, paraît actif dès la formation de l'ensemble linguistique proto-océanique (François, 2004). Ce système comprend un axe cardinal ouest/est ou nord-ouest/sud-est (François, 2004) fixe, utilisé en navigation, et un système orthogonal d'orientation, relatif, opérant à l'échelle locale, qui combine l'axe terre-mer et l'axe parallèle au rivage (Ozanne-Rivierre, 1997). L'application aux sépultures étudiées par José Garanger montre que si l'axe cardinal, plus général, n'intéresse que peu de sépultures (de 9 à 16 sur un total de 112), le système orthogonal local, en revanche, s'applique à toutes. Les sépultures sont en majorité orientées selon l'axe terre-mer, avec la tête côté terre. Selon cette grille de lecture, les différences remarquées par José Garanger entre Efate et Tongoa ne sont plus perceptibles, suggérant l'adhésion des groupes installés sur ces îles au même système océanien de représentation de l'espace.

\section{Traitement du squelette}

Les inhumations que décrit et illustre José Garanger correspondent au dernier traitement du corps ; elles peuvent être qualifiées de définitives. Les 71 squelettes en situation primaire entièrement mis au jour sont virtuellement complets. Ils n'ont manifestement pas fait l'objet de prélèvement d'ossements. Toutefois, une autre forme de traitement du défunt, impliquant une manipulation de ses ossements, a dû exister durant le deuxième millénaire après J.-C. La pratique est suggérée par la présence de 13 dépôts secondaires d'ossements dans trois sites de la région Efate-Shepherd. Les modalités de mise œuvre de ce traitement sont délicates à préciser. Les données disponibles indiquent que les défunts impliqués n'auraient pas été sélectionnés en fonction de leur âge au décès et que si la mise en terre du cadavre était une option possible pour obtenir les ossements, elle a été peu utilisée, car aucune trace d'exhumation n'a été décelée par José Garanger.

Ces dépôts d'ossements se caractérisent par la diversité de leur composition anatomique et par la similitude de leur localisation dans l'espace sépulcral (tableau 4). Ils apparaissent régulièrement en étroite association avec une inhumation primaire. Les agencements relatifs des dépôts primaires et secondaires - absence de modification ou perturbation de l'inhumation primaire, similitude des dispositions et orientation - suggèrent leur simultanéité : les ossements ont été placés dans la sépulture en même temps que le cadavre.

\section{Il y a trois mille ans à Teouma (Efate)}

Localisé sur la côte sud de l'île d'Efate (figure 1), le site Lapita de Teouma a été fortuitement découvert en 2004 au cours de travaux d'extraction de sable (Bedford et al., 2004, 2006). Il se trouve aujourd'hui à environ $800 \mathrm{~m}$ du bord de mer, enfoui sous plus de $80 \mathrm{~cm}$ de sédiment. Les recherches archéologiques révèlent que la première occupation du site était de nature exclusivement funéraire (figure 2). Le cimetière de Teouma a été implanté sur l'ancienne plage formée de blocailles de corail ainsi que dans les cavités et à la surface du récif corallien surélevé la bordant. Ces zones avaient été recouvertes et remplies par des cendres volcaniques peu de temps avant l'utilisation du lieu par la communauté inhumante.

L'étroite relation entre ce cimetière et le peuplement initial du Vanuatu est clairement démontrée par : l'association des sépultures avec un ensemble de poteries Lapita (Bedford et Spriggs, 2007 ; Bedford et al., 2007) ; la datation directe des squelettes révélant des décès survenus autour de 1050 ans avant J.-C. (Spriggs et al., en prép); et des observations stratigraphiques montrant le recouvrement de l'horizon sépulcral par une couche culturelle, d'environ $50 \mathrm{~cm}$ d'épaisseur, résultant d'activités domestiques rapportées à la phase Erueti ancien datée d'environ 750-450 ans avant J.-C. (Bedford et al., 2004 ; 2006 ; sous presse).

Plusieurs indices suggèrent que l'utilisation du cimetière s'est étalée dans le temps : le bris et la dispersion de poteries au cours des activités mortuaires, la superposition d'inhumations, et la 


\begin{tabular}{|c|c|c|c|c|c|}
\hline Composition & Forme & Structuration & Localisation & $\begin{array}{l}\text { Nombre de } \\
\text { cas et site }\end{array}$ & Âge au décès \\
\hline $\begin{array}{l}1 \text { squelette } \\
\text { virtuellement } \\
\text { complet }\end{array}$ & $\begin{array}{l}\text { «paquet» } \\
\text { parallélé- } \\
\text { pipédique }\end{array}$ & $\begin{array}{l}\text { ossements réarrangés } \\
\text { avec le crâne à une extré- } \\
\text { mité, le bassin à l'autre } \\
\text { et le reste des os au } \\
\text { milieu }\end{array}$ & $\begin{array}{l}1 \text { placé entre les membres } \\
\text { inférieurs d'un corps } \\
\text { inhumé sur le dos, } \\
2 \text { placés parallèlement à un } \\
\text { corps, presque à son } \\
\text { contact, avec une } \\
\text { orientation identique à celle } \\
\text { des dépôts primaires en } \\
\text { couple }\end{array}$ & $\begin{array}{l}5 \text { cas à Retoka } \\
\text { dont } 2 \text { sont } \\
\text { des nouveau-nés }\end{array}$ & $\begin{array}{l}\text { adulte ou } \\
\text { nouveau-né }\end{array}$ \\
\hline $\begin{array}{l}1 \text { squelette } \\
\text { virtuellement } \\
\text { complet }\end{array}$ & $\begin{array}{l}\text { «paquet» } \\
\text { parallélé- } \\
\text { pipédique }\end{array}$ & $\begin{array}{l}\text { fagot sur lequel le crâne } \\
\text { avait été posé }\end{array}$ & $\begin{array}{l}\text { placé parallèlement à une } \\
\text { inhumation primaire }\end{array}$ & $\begin{array}{l}1 \text { cas à } \\
\text { Mangarisu } \\
(\text { TO 28-3) }\end{array}$ & adulte? \\
\hline $\begin{array}{l}1 \text { bloc } \\
\text { cranio-facial } \\
\text { ou } 1 \\
\text { mandibule }\end{array}$ & - & - & $\begin{array}{l}\text { sur la cuisse } \\
\text { à côté de la tête ou de la } \\
\text { main d'une inhumation } \\
\text { primaire }\end{array}$ & $\begin{array}{l}1 \text { cas à Retoka } \\
3 \text { cas à } \\
\text { Mangarisu } \\
(\text { TO 28-3) }\end{array}$ & adulte? \\
\hline des os longs & «fagot » & - & $\begin{array}{l}\text { à proximité des inhumations } \\
\text { primaires en couple }\end{array}$ & 1 cas à Retoka & 6 adultes \\
\hline $\begin{array}{l}\text { des } \\
\text { fragments de } \\
\text { crânes, coxaux et os } \\
\text { longs }\end{array}$ & amas & $\begin{array}{l}\text { pas de structuration } \\
\text { décelée }\end{array}$ & sur la poitrine & $\begin{array}{l}1 \text { cas sur le } \\
\text { site TO 29-7 } \\
\text { Mangarisu }\end{array}$ & $\begin{array}{l}\text { représentant } \\
1 \text { adulte et } \\
1 \text { enfant }\end{array}$ \\
\hline $\begin{array}{l}\text { ossements } \\
\text { brisés }\end{array}$ & amas & $\begin{array}{l}\text { pas de structuration } \\
\text { décelée }\end{array}$ & sur la poitrine & $\begin{array}{l}1 \text { cas sur le } \\
\text { site de Ti Tongoa } \\
\text { Liseiriki }\end{array}$ & $?$ \\
\hline
\end{tabular}

TABLEAU 4. - Inventaire et description des dépôts secondaires d'ossements mis au jour par José Garanger (1972)

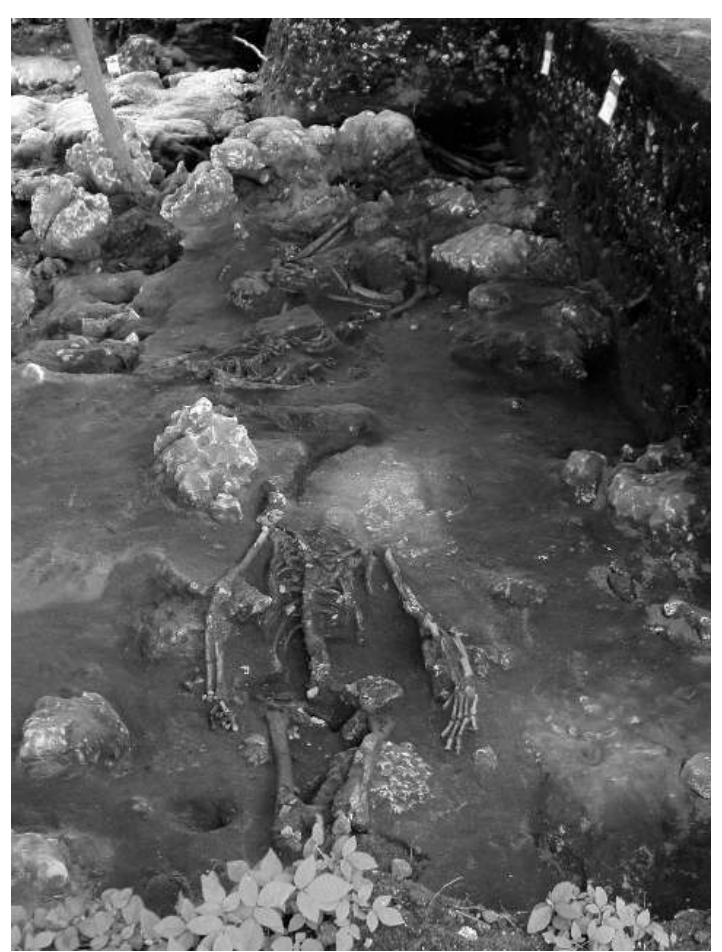

Figure 2. - Vue générale du cimetière Lapita de Teouma (Efate)

procédure funéraire elle-même (Valentin et al., en préparation).
Trois campagnes de terrain (2004-2005-2006) ont permis de reconnaitre quarante-huit faits funéraires se subdivisant en cinquante-et-une entités sépulcrales distinctes, et des os épars dispersés en différents points du remplissage (Bedford et al., sous presse). Ces entités se répartissent en 6 inhumations de nouveau-nés ou fœtus, 34 inhumations d'adultes - hommes et femmes et 11 dépôts secondaires renfermant les restes d'un ou plusieurs sujets. Les résultats d'une analyse de l'état de santé des individus sont présentés par Hallie Buckley (2007) et Buckley et al. (2008) et la question de l'origine géographique (locale ou étrangère) des défunts a été abordée par Alexander Bentley et collaborateurs (2007) par le biais d'analyses isotopiques du strontium.

\section{Traitement du corps}

L'inhumation semble le procédé préférentiellement employé. Quarante inhumations sont actuellement recensées contre une possible incinération (Fuller, 2006). Cette dernière pratique a dû être limitée, même s'il demeure difficile d'en évaluer l'extension en raison, d'une part, de facteurs taphonomiques tels les remaniements engendrés par l'occupation Erueti et les pertes causées par les travaux d'extraction de sable 
et, d'autre part, de la fouille incomplète du cimetière.

\section{Position initiale du corps}

À Teouma, les corps ont été déposés souvent sur le dos $(\mathrm{N}=23)$, parfois sur le ventre $(\mathrm{N}=8)$, avec les membres dans des positions très variées (figure 3 ). Les individus en complète extension sont peu nombreux ( 7 contre 24$)$. Les bras sont généralement le long du corps $(\mathrm{N}=39)$, les coudes sont fréquemment étendus $(\mathrm{N}=19)$, plus rarement fléchis $(\mathrm{N}=7)$. Les membres inférieurs montrent une nette tendance à la flexion, voire à l'hyperflexion, des hanches et des genoux ( 15 cuisses fléchies sur 54 et 30 genoux fléchis sur 45). Dans trois sépultures, les jambes étaient croisées.
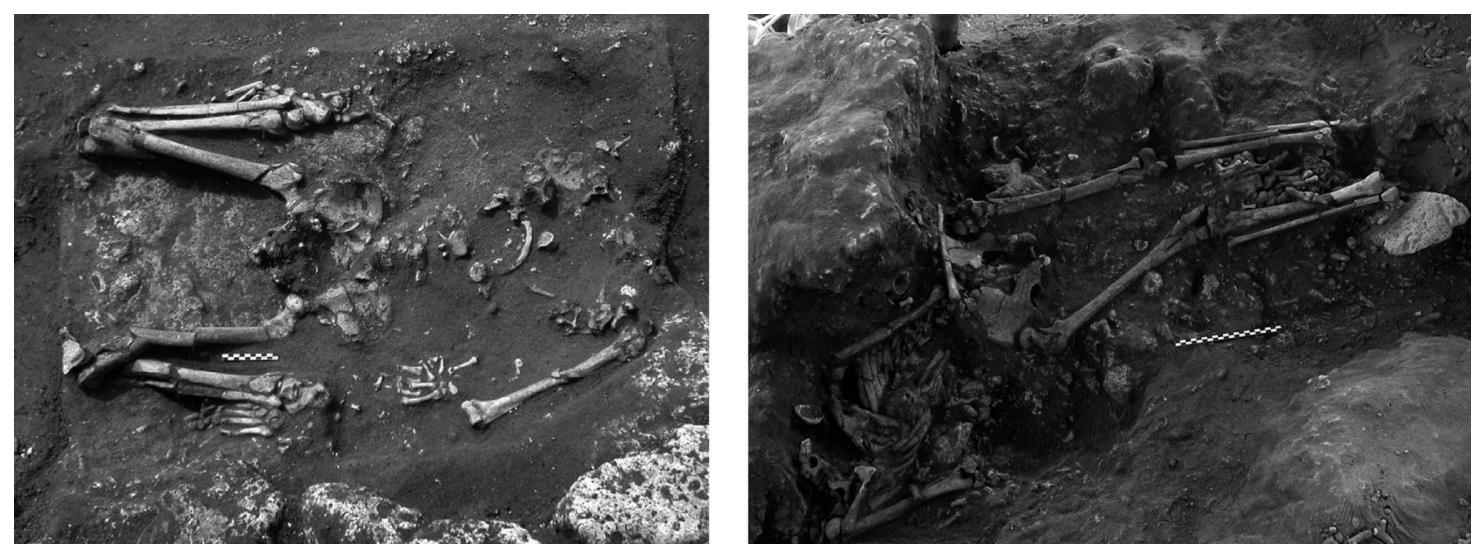

Figure 3. - Exemples de position d'inhumation dans le cimetière Lapita de Teouma (Efate) Un cas de décubitus dorsal (B25) et un cas de décubitus ventral (B47).

Noter les importants manques osseux.

\section{Parure et mobilier}

Des anneaux en Conus ont été découverts dans plusieurs inhumations, généralement à l'endroit où le crâne aurait dû être. Toutefois les défunts semblent n'avoir été que rarement parés pour leur enterrement ( 1 sur 40 portait deux bracelets) alors que des objets de parure (bracelets en Conus, et Trochus, anneaux et perles en Conus) sont présents dans les assemblages Lapita du Vanuatu (Bedford, 2006), notamment dans celui recueilli sur le site de Teouma.

Le mobilier funéraire est peu varié et peu abondant. Des poteries décorées de motifs pointillés et des valves non modifiées d'un gros coquillage de mangrove (Geloina coaxans) avaient été associées à certains défunts.

\section{Sépultures multiples}

Les travaux menés jusqu'à présent sur le site de Teouma n'ont pas clairement mis en évidence le dépôt simultané de plusieurs corps.

\section{Orientation}

Les inhumations de Teouma se caractérisent par la diversité des orientations (tableau 5). Il n'apparaît pas de prépondérance de l'un des quatre axes sur l'autre et de l'une des huit directions sur l'autre, ni d'une direction sur l'autre pour un même axe.

Ces observations incitent à penser que la répartition spatiale actuellement observée n'est pas directement structurée par le système cardi-

\begin{tabular}{|l|c|c|c|c|c|c|c|c|}
\hline Site & SE/NW & NW/SE & W/E & E/W & NE/SW & SW/NE & N/S & S/N \\
\hline Teouma & 3 & 1 & 5 & $\begin{array}{c}7 \\
\text { (dont } 1 \mathrm{NN})\end{array}$ & 4 & 3 & 3 & $\begin{array}{c}6 \\
(\text { dont } 1 \mathrm{NN})\end{array}$ \\
\hline Fréquence & $9,4 \%$ & 3,1 & 15,6 & 21,0 & 12,5 & $9,4 \%$ & $9,4 \%$ & $18,7 \%$ \\
\hline
\end{tabular}

TABLeAu 5. - Orientation des sépultures dans le cimetière Lapita de Teouma (Efate) 
nal d'orientation. Les absences de représentation massive d'une direction par rapport à l'autre pour un axe donné et d'une orientation privilégiée dans la zone de plage suggèrent que cette distribution spatiale pourrait, au moins en partie, avoir été influencée par des facteurs naturels comme la forme des cavités utilisées pour déposer certains corps.

Il existe un parallèle entre l'orientation la plus fréquemment recensée à Teouma et l'axe cardinal du système océanien d'orientation (axe ouest/est). Cet axe est aussi celui présenté par les trois dépôts secondaires orientables. Identifier un axe terre-mer est délicat en raison de la configuration compliquée de la baie de Teouma. Toutefois, l'orientation nord/sud, qui constitue l'axe de la vallée et de la baie elle-même, aurait pu avoir été privilégiée. Cet axe nord/sud est le second en termes de fréquence d'apparition.

\section{Traitement du squelette}

À Teouma, l'inhumation ne représentait pas la fin du traitement du défunt. Après un laps de temps durant lequel le cadavre s'est décomposé, des membres de la communauté Lapita sont retournés à la tombe et ont prélevé des ossements, principalement dans la moitié supérieure du squelette (figure 3). Les crânes sont constamment absents, les radius et ulnas le sont fréquemment, les clavicules, les scapulas, les humérus, les côtes le sont aussi parfois. Toutes les sépultures adultes découvertes sont, selon la terminologie de Duday et al. (1990), des inhumations incomplètes. Le dépôt initial d'individus plus complets est en effet attesté par la présence, dans la majorité des cas, de dents isolées, d'os hyoïde, et d'os des mains, alors que la réalité du prélèvement après décomposition dans la tombe est confirmée par la présence in situ de petits os, l'absence de tri horizontal ou vertical des os et artéfacts qui aurait pu suggérer un phénomène taphonomique, et la répétition des mêmes absences d'une sépulture à l'autre.

Cette façon complexe de traiter le défunt se rapproche de celle initialement décrite par Robert Hertz (1907) sous le nom de «doubles funérailles ». De multiples exemples en sont ethnographiquement connus en Mélanésie et, en particulier, dans le nord du Vanuatu (par exemple: Deacon, 1934 ; Layard, 1934 ; Speiser, 1996).

La découverte de dépôts secondaires dans le cimetière de Teouma indique de plus l'existence d'une forme particulière de traitement des ossements humains en relation avec l'espace sépulcral. Onze dépôts secondaires ont actuellement

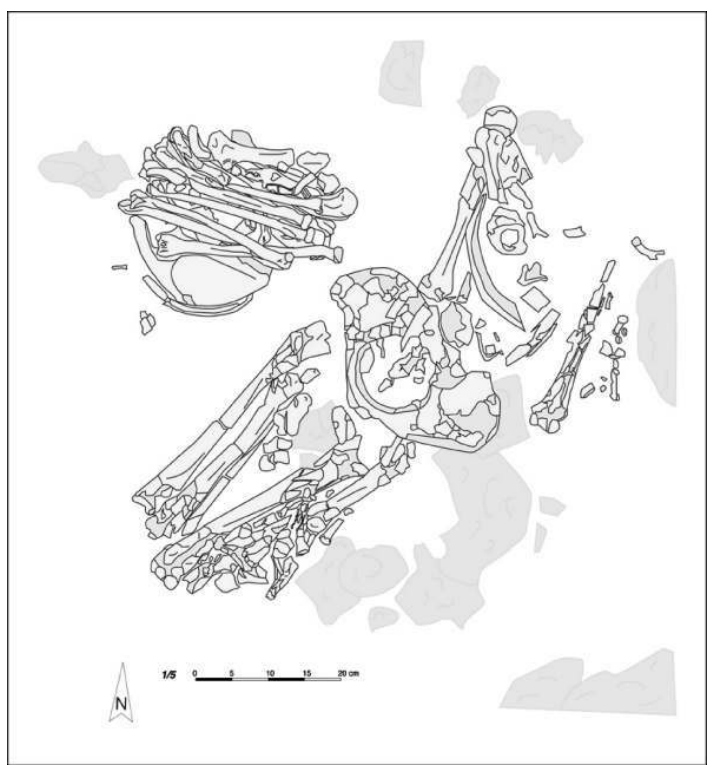

Figure 4. - Exemple d'un corps déposé en position hyperfléchie forcée (B33) et d'un dépôt secondaire d'ossements formé principalement par une pile d'os des avantbras (B39) (relevé Fidel Yoringmal, mise au net Mikael Michelland)

été mis au jour sur le site. Ils sont généralement formés d'os adultes représentant de 1 à 4 individus au moins, des os immatures (1 nouveau-né et 1 enfant d'environ 10 ans) ayant été observés dans un cas. La présence de sépultures incomplètes suggère qu'au moins certaines d'entre elles ont pu être la source des os redéposés au cimetière.

Ces dépôts se caractérisent par la diversité à la fois de leur composition anatomique et de leur structuration spatiale : alignement de trois blocs crâniofaciaux entourés de blocs de corail ; crâne posé sur un large bracelet en Conus dans une jarre fermée par un plat; restes infracrâniens déposés dans des poteries, alignement de trois mandibules sur lequel des os longs et des coxaux ont été posés ; pile formée de trois blocs crâniofaciaux, d'une mandibule et de restes infracrâniens ; pile d'os des avant-bras et de la partie supérieure du thorax posés sur des scapula; assemblages de restes infracrâniens (figure 4 et figures in Bedford et al., 2006, 2007, sous presse).

Ces dépôts secondaires étaient à proximité des sépultures incomplètes. Ainsi, la pile d'os des avant-bras a été mise au jour à $10 \mathrm{~cm}$ environ d'une inhumation reposant sur le dos avec les genoux en hyperflexion forcée (figure 4). L'association entre les deux types de dépôt est parfois très étroite. Par exemple, l'alignement de trois blocs crânio-faciaux reposait sur le haut de la cage thoracique d'un individu en décubitus dorsal. Dans ce cas, les dépôts ont été successifs. 
L'indiquent les modifications (dislocation et prélèvement) altérant l'ordonnancement et l'intégrité du squelette sous-jacent. La mise en place du dépôt secondaire s'est effectuée sur le squelette incomplet, et non sur le corps du défunt.

\section{Une comparaison qualitative : quelques ressem- blances mais surtout des différences}

L'inhumation apparaît comme le moyen préférentiellement utilisé pour faire disparaître le corps du défunt il y trois mille ans à Teouma et dans les sites du deuxième millénaire après J.-C. étudiés par José Garanger. C'est aussi le traitement reflété par les sépultures de la fin du premier millénaire avant J.-C. de Mele (Taplins) (Ward et Houghton, 1988, 1991) et par celles rapportées aux derniers 1000 ans découvertes par d'autres archéologues à Mangaliliu (Ouest Efate) (Valentin et al., 2005) et à l'îlot Fila (Sud Efate), à Tanna et Futuna (Shutler et al., 2002) ainsi qu'à Aneityum (Spriggs, 1997 : 217-218). L'incinération, traitement probablement rare à Teouma, ne semble pas avoir été pratiquée au cours du dernier millénaire dans la région EfateShepherd et, d'une façon plus générale, dans les autres régions de l'archipel ayant fait l'objet de recherches archéologiques, mais les vestiges en sont fugaces et archéologiquement difficiles à détecter. De plus, d'autres types de traitement du corps, comme l'exposition du cadavre, ont dû exister dans la région durant cette période, mais ils demeurent difficiles à caractériser, faute d'une documentation archéologique appropriée.

Dans le détail, la comparaison des cinq composantes du dispositif funéraire observées ici met en lumière de nombreuses différences entre sépultures anciennes et récentes, même si la documentation disponible est lacunaire et inégalement répartie sur les trois mille ans de préhistoire.

Ainsi, les types de posture et de flexion des membres choisis distinguent clairement les deux groupes. Une plus grande diversité des positions ainsi que des fréquences plus élevées d'hyperflexion des membres et de corps déposés sur le ventre opposent les sépultures de Teouma aux sépultures plus récentes, caractérisées par une relative uniformité des positions, les corps étant souvent allongés sur le dos, membres étendus. L'extension du corps est aussi une attitude souvent choisie (3/4) dans le site de la fin du premier millénaire avant J.-C. de Mele (Taplins) (Ward and Houghton, 1988, 1991).

L'usage de parure funéraire offre aussi des différences claires entre les deux ensembles. La pratique est rare à Teouma. Elle apparaît rare également dans les sépultures de la fin du premier millénaire avant J.-C. de Mele (Taplins 1), où des perles discoïdes ou "monnaies" et un fragment d'ornement en Conus ont été mis au jour, alors qu'elle est presque constante dans les sites du deuxième millénaire après J.-C. d'Efate (Mele, Lelepa Mangaasi, Retoka, et Fila), régulière à Futuna et Aneityum et peu fréquente à Tongoa.

Des distinctions dans les modes de traitement du squelette après décomposition du corps sont également manifestes. L'inhumation des adultes était temporaire à Teouma, alors qu'elle était définitive dans les sépultures individuelles et multiples des premier et deuxième millénaires après J.-C. de la région Efate-Shepherd. Cette dernière pratique semble aussi avoir été dominante dans le sud de l'archipel, un unique cas d'absence du crâne étant décrit dans la sépulture multiple d'Anelcauhat, à Aneityum, datée de 1500-1600 ans après J.-C. (Spriggs, 1997 : 217218). Le traitement des ossements montre par ailleurs une convergence de forme : des dépôts secondaires ont été réalisés dans l'espace sépulcral, à proximité ou en étroite association avec les inhumations au début et à la fin de la préhistoire. Pourtant, ce comportement en apparence analogue relève de logiques différentes, ayant une relation avec le caractère définitif ou non de l'inhumation : à Teouma, le dépôt a dans certains cas été associé au squelette alors qu'à Retoka et à Mangarisu, il a été associé au cadavre.

L'absence de sépultures multiples constituerait, dans l'état actuel des connaissances, une autre différence entre les sépultures Lapita et les plus récentes, la sépulture d'Anelcauhat (Aneityum) (Spriggs, 1997 : 217-218) complétant l'inventaire des sépultures multiples du deuxième millénaire après J.-C.. Indiquant l'ancienneté de la pratique sur Efate, une possible sépulture double a été identifiée à Mele (Taplins), qui présente la mise en scène originale d'un corps masculin et d'un corps féminin (Ward and Houghton, 1988). Les défunts avaient été placés au contact l'un de l'autre, tête-bêche avec la même orientation nord-ouest/sud-est, la femme en position hyperfléchie, genoux ramenés contre la poitrine et l'homme allongé sur le dos avec une poterie complète à côté de la tête.

La comparaison des orientations enregistrées selon le système cardinal montre des différences entre les sépultures de Teouma et les sépultures de l'îlot Mele, Lelepa et Retoka caractérisée par une prédominance de l'orientation sud-ouest/ nord-est. Les orientations utilisées à Teouma se retrouvent en revanche à Tongoa et dans les sites 
du sud de l'archipel (Tanna, Futuna) ainsi que dans les sépultures de la fin du premier millénaire avant J.-C. de Mele (Taplins). Cette convergence pourrait être due au hasard, les corps ayant été inhumés dans des abris sous-roche dont la morphologie a pu influer sur l'orientation des dépôts. Lorsque le système océanien d'orientation est utilisé, les variations s'estompent, mais la différence entre Teouma et les autres sites de la région Efate-Shepherd subsiste : l'axe cardinal suivi de l'axe terre-mer sont les orientations dominantes à Teouma, alors que toutes les sépultures du deuxième millénaire après J.-C. s'orientent suivant les axes locaux d'orientation, avec une dominance de l'axe terre-mer.

Finalement, le seul point commun entre les sépultures anciennes et récentes d'Efate est peutêtre la présence assez fréquente de dépôts de coquillages non modifiés près des défunts (à Mangaasi et Retoka). Une pratique qui apparaît également dans les sépultures de la fin du premier millénaire avant J.-C. de Mele (Taplins 1). L'association de poterie avec les défunts marque une autre ressemblance entre ce dernier site et celui de Teouma. Mais de semblables dépôts ne sont pas mentionnés dans les sépultures du sud de l'archipel datant des derniers mille ans.

\section{Une évolution des mentalités ou des mentalités différentes?}

\section{Un changement du dispositif funéraire?}

Les différences observées pourraient suggérer un changement radical de système funéraire entre le début du premier millénaire avant J.-C. et les périodes suivantes de la chronologie préhistorique. Toutefois, unique, le site de Teouma pourrait aussi, malgré la cinquantaine de sépultures mises au jour, ne refléter qu'une facette des pratiques funéraires Lapita. Par ailleurs, les quatre tombes de Mele (Taplins) (Ward and Houghton, 1988) indiquent que la pratique d'une inhumation définitive, l'utilisation de sépulture multiple d'adultes et peut-être de la position étendue, faisait partie de la panoplie funéraire d'Efate, à la fin du premier millénaire avant J.-C., au moment où l'on commençait à fabriquer les poteries de type Mangaasi dans la région EfateShepherd (Bedford, 2006). La différence observée entre ce site et Teouma serait-elle l'indice d'une diversification des pratiques funéraires faisant écho au phénomène régional de diversification locale opérant dès la fin de la fabrication de la poterie Lapita qui, dans le centre du Vanuatu, a produit les poteries de type Erueti puis Man- gaasi (Bedford, 2006; Bedford and Clark, 2001)? Tandis que l'association de poterie aux défunts, observée à Mele (Taplins) comme à Teouma, constituerait dans ce schéma un exemple local d'une persistance d'une pratique ancienne ayant pu exister à l'échelle régionale. Plusieurs exemples d'association entre sépultures et poteries ont en effet été recensés (Bedford and Spriggs, 2007) dont celui d'une poterie de type Podtanean (fin de la période Lapita) couvrant le crâne du défunt dans une sépulture du site de Lapita en Nouvelle-Calédonie (Valentin et Sand, 2000). Il est délicat de l'affirmer face à une documentation archéologique encore insuffisante. Un changement plus récent, vers 1000 ans après J.-C., est de même difficile à identifier. Les "perdurations" des pratiques d'une ornementation limitée du défunt à Tongoa et d'un dépôt de coquilles non travaillées avec le corps à Efate (Retoka et Mangaasi) correspondent-elles à de véritables continuités historiques ou à des convergences comportementales ? Il convient en effet de s'interroger sur la nature même des similitudes entre les ensembles de sépultures car il existe une lacune documentaire s'étalant, dans l'état actuel des connaissances, sur 1000 à 1500 ans.

\section{Des influences orientales?}

La présence d'objets de parure d'affinité polynésienne identifiés par José Garanger dans plusieurs sépultures d'Efate, ainsi que par d'autres dans des sépultures d'Aneityum (Spriggs, 1997 : 217-218) et de Futuna (Shutler et al., 2002), pourrait résulter de l'action du vaste système d'interactions aujourd'hui évoqué par les archéologues pour expliquer le changement majeur de la culture matérielle survenu il y a mille ans dans la région Efate-Shepherd (Bedford, 2006 : 217). Ces influences polynésiennes ont-elles, au-delà de l'ornementation du défunt, façonné les pratiques funéraires à l'œuvre dans la région durant le deuxième millénaire après J.-C. ? L'association d'inhumations animales et humaines observées dans certains sites de Polynésie orientale (par exemple, à Manihina, aux Marquises, où cochons et chiens ont été inhumés avec les humains (Conte, 2002)) et l'association de dépôts secondaires et primaires constatée dans plusieurs sites de Polynésie occidentale, à 'Atele, Tonga (Davidson, 1969) et Petania, Wallis (Sand and Valentin, 1991) et orientale, à Manihina, Marquises (Conte, 2002; Maureille et Sellier, 1996), pourraient-elles en être l'indice, en dépit de la distance séparant les îles? 
La comparaison avec des exemples archéologiques actuellement connus dans la littérature montre toutefois qu'il n'y a pas de parallèles directs entre les sépultures de la région EfateShepherd et celles de Polynésie occidentale, les tombes présentant des architectures et des localisations spatiales très différentes. Mais une forme d'influence idéologique pourrait peut-être être évoquée, de possibles analogies existant entre "la démonstration de pouvoir » reflétée par l'ensemble sépulcral de Retoka (Garanger, 1972 ; Testart, 2004 ; contra Luders, 2001) et les tombes de chefs tongiens (Kirch, 1988 ; Sand et $a l ., 2006)$. On rejoindrait là l'idée émise par Bedford et Spriggs (2008: 110) pour définir la nature $\mathrm{du}$ contact polynésien dans le centre du Vanuatu :

« Neither conquest nor major migratory events need be invoked necessarily to explain conscious or unconscious processes of imitation, adaptation, or inclusion of exotic cultural practices and material culture »

alors qu'une interaction plus soutenue, incluant une composante migratoire, a pu se produire dans certaines régions du centre et du sud de l'archipel (Mele, Fila, Futuna).

L'hypothèse d'interactions avec l'archipel fidjien pourrait aussi être avancée, le dépôt de morts d'accompagnement dans la tombe apparaissant comme une composante récurrente du rituel funéraire fidjien ancien variant avec le rang social du défunt (Valentin et al., 2007). En effet, les descriptions ${ }^{1}$ tant ethnohistoriques qu'archéologiques (par exemple Schütz, 1977 : 152; Williams, 1858: 189; Rechtman, 1992 : 170, fig. A19) rappellent bien les situations observées dans les sépultures multiples de Retoka, Mangarisu et Aneityum. La similitude des pratiques pourrait peut-être témoigner d'une interaction entre les deux archipels au moins durant le deuxième millénaire après J.-C. Une interaction également marquée par la présence de formes de poterie particulières et de rituels associés, tant à Fidji que dans le nord du Vanuatu à la fin de la préhistoire (Bedford, 2006 ; Bedford and Spriggs, 2008).

$\mathrm{Au}$ final, cette analyse met en lumière la grande diversité des gestes et comportements funéraires mis en œuvre durant les trois mille ans de préhistoire du Vanuatu. Leurs dynamiques historiques demeurent, en revanche, encore hypothétiques. Nous espérons que notre approche des sépultures préhistoriques de la région Efate-Shepherd ne trahit pas le message scientifique qu'a laissé José Garanger :

« traiter les questions archéologiques en ethnologue ; considérer les résultats obtenus non comme définitifs mais comme relatifs; se défier des généralisations, l'interprétation des cultures préhistoriques étant toujours plus complexe qu'on ne le suppose. " (Robineau, $2007: 343$ )

\section{Remerciements}

Nous remercions vivement Christophe Sand pour son invitation à contribuer à ce numéro en hommage à José Garanger, pionnier de la préhistoire du Vanuatu. Nous souhaitons également adresser nos remerciements aux membres du Centre culturel du Vanuatu, à la famille Monvoisin et à la population d'Eratap qui nous ont aidé à mener à bien les recherches archéologiques sur le site de Teouma ainsi qu'à Maurice Hardy pour son assistance dans la réalisation des illustrations. Et, en particulier, Frédérique Valentin remercie très sincèrement José et Suzy Garanger qui ont généreusement mis à sa disposition leur documentation sur les sépultures du Vanuatu.

\section{BIBLIOGRAPHIE}

Albert Jean-Pierre, Éric CRUBÉzy et Béatrix Midant-Reynes, 2000. L'archéologie du sacrifice humain. Problèmes et hypothèses. Séminaire du Centre d'Anthropologie de Toulouse, Archéo-Nil 10, pp. 9-18.

BEDFord Stuart, 2006. Pieces of the Vanuatu Puzzle: Archaeology of the North, South and Centre, Canberra, Pandanus Press, Australian National University, Terra Australis 23, (http://epress.anu.edu. au/ta23_citation.html).

BEDFORD Stuart and Geoffrey Clark, 2001. The rise and rise of the incised and applied relief tradition: A review and reassessment, in G.R. Clark, A.J.

1. Au XIX ${ }^{\mathrm{e}}$ siècle à Fidji, l'inhumation du défunt se déroulait de la façon suivante : «The body of the chief is then deposited in its resting place, and a strangled female is placed at each side. The one on the right side of the chief has her right hand laid on his breast, while she on the left has her left » (Schütz, $1977: 152)$. Et « The bodies of these victims are called « grass » for bedding the Chief's grave » (Williams, 1858 : 189). Révélant l'ancienneté de la pratique, des exemples archéologiques pré-européens ont notamment été découverts dans l'île de Wakaya (Sud-Ouest Fidji) (Rechtman, 1992). Ainsi, comprise parmi un ensemble de 23, une sépulture triple donne à voir une mise en scène remarquable : au fond d'une fosse, deux femmes avaient été disposées selon une symétrie en miroir ; elles gisaient sur le dos, leur face et leurs pieds se faisant face, les membres supérieurs fléchis, les membres inférieurs fléchis latéralement à droite pour l'une et à gauche pour l'autre ; le corps d'un homme avait été déposé sur elles, au centre de la sépulture, allongé sur le dos, les membres supérieurs écartés du corps et fléchis, les mains à hauteur du bassin, les membres inférieurs présentant une flexion latérale gauche (Rechtman, 1992 : 170, fig. A19). 
Anderson and T. Vunidilo (eds), The archaeology of Lapita dispersal in Oceania, Canberra, Australian National University E Press, Terra Australis 17, pp. 61-74.

Bedford Stuart, Andrew Hoffman, Marta Kaltal, Ralph Regenvanu and Richard Shing, 2004. Dentate-stamped Lapita reappears on Efate, Central Vanuatu: a four decade-long drought is broken, Archaeology in New Zealand 47 (1), pp. 39-49.

BEDFORD Stuart and Matthew Spriggs, 2007. Birds on the rim: a unique Lapita carinated vessel in its wider context, Archaeology in Oceania 42, pp. 12-21.

Bedford Stuart and Matthew Spriggs, 2008. Northern Vanuatu as a Pacific Crossroads: The Archaeology of Discovery, Interaction, and the Emergence of the "Ethnographic Present", Asian Perspectives 47 (1), pp. 95-120.

BeDFord Stuart, Matthew Spriggs, Hallie Buckley, Frédérique VALENTIN and Ralph REgENVANu, sous presse. The Teouma Lapita site, South Efate, Vanuatu: a summary of three field seasons (20042006), in P. Sheppard, T. Thomas and G. Summerhayes (eds), Lapita Conference 2007, Honiara, to be published in NZAA monograph series 2009.

BEDFORD Stuart, Matthew Spriggs and Ralph REgENVANU, 2006. The Teouma Lapita site and the early human settlement of the Pacific Islands, Antiquity 80, pp. 812-828.

Bedford Stuart, Matthew Spriggs, Ralph Regenvanu, Colin Macgregor, Takaronga Kuautonga and Michael SIETz, 2007. The excavation, conservation and reconstruction of Lapita burial pots from the Teouma site, Efate, Central Vanuatu, in S. Bedford, C. Sand and S. Connaughton (eds), Oceanic Explorations: Lapita and Western Pacific Settlement, Canberra, Australian National University E Press, Terra Australis 25, pp. 223-240.

BeDFord Stuart, Matthew Spriggs, Meredith WiLson and Ralph Regenvanu, 1998. The Australian National University-National Museum of Vanuatu Archaeology Project, 1994-7: A Preliminary Report on the Establishment of Cultural Sequences and Rock Art Research, Asian Perspectives 37 (2), pp. 165-193.

Bentley Alexander, Hallie Buckley, Matthew Spriggs, Stuart Bedford, Chris. J. Ottley, Geoff M. Nowell, Colin G. Macpherson and D. Graham Pearson, 2007. Lapita Migrants in the Pacific's Oldest Cemetery: Isotopic Analysis at Teouma, Vanuatu, American Antiquity 72 (4), pp. 645-656.

Buckley Hallie, 2007. Possible Gouty Arthritis in Lapita-Associated Skeletons from Teouma, Efate Island, Central Vanuatu, Current Anthropology 48 (5), pp. 741-749.

Buckley Hallie, Nancy Tayles, Matthew Spriggs and Stuart BEDFORD, 2008. A preliminary report on health and disease in early Lapita skeletons: Possible biological costs of colonization, Journal of Island and Coastal Archaeology 3 (1), pp. 87-114.
Codrington Robert Henry, 1885. The Melanesian languages. Oxford, Clarendon Press.

CONTE Éric, 2002. Current research on the Island of Ua Huka, Marquesas Archipelago, French Polynesia, Asian Perspectives 41 (2), pp. 258-268.

DAvidson Janet, 1969. Archaeological excavation at two burial mounds at 'Atele, Tongatapu, Records of the Auckland Institute and Museum 6 (4-6), pp. 251286.

DEACON Bernard, 1934. Malekula, a vanishing people in the New Hebrides, (edited by C.H. Wedgwood), London, Routledge.

Duday Henri, Patrice Courtaud, Éric Crubézy, Pascal SELliER et Anne-marie TILLIER, 1990. L'anthropologie « de terrain » : reconnaissance et interprétation de gestes funéraires, Bulletins et mémoires de la Société d'Anthropologie de Paris 2 (3-4), pp. 29 50.

Espirat J.J., J. Guiart, M.S. Lagrange et M. Renaud, 1973. Système des titres dans les Nouvelles-Hébrides centrales, d'Efate aux îles Shepherd, Paris, Institut d'Ethnologie, Muséum national d'histoire naturelle, musée de l'Homme, Mémoires de l'Institut d'Ethnologie.

FrançOIS Alexandre, 2004. Reconstructing the Geocentric System of Proto-Oceanic, Oceanic Linguistics 43 (1), pp. 1-31.

FULler Rachel, 2006. A study of the non-burial human bone from the Lapita site of Teouma, Vanuatu, Thesis submitted in partial fulfilment for the Post Graduate Diploma of Science, University of Otago, New Zealand.

Garanger José, 1972. Archéologie des NouvellesHébrides : contribution à la connaissance des îles du centre, Paris, Publications de la Société des Océanistes 30 .

_, 1997. Oral tradition and archaeology: two cases from Vanuatu, in R. Blench and M. Spriggs (eds), Archaeology and Language I, Theoretical and Methodological Orientations, Londres, Routledge, One World Archaeology 27, pp. 321-330.

Hertz Robert, 1907. Contribution à une étude sur la représentation collective de la mort, L'année sociologique X, pp. 43-137.

KIRCH Patrick Vinton, 1988. Niuatoputapu. The prehistory of a Polynesian Chiefdom, Washington State Museum, Thomas Burke Memorial, Monograph 5.

LAYARD John, 1934. The journey of the dead, in E. E. Evans-Pritchard, R. Firth, B. Malinowski and I. Shapera (eds), Essays presented to C. G. Seligman, London, Kegan Paul, Trench, Trubner and co, pp. 113-142.

LuDERs David, 2001. Retoka revisited and Roimata revised, The Journal of the Polynesian Society 110 (3), pp. 247-287.

Maureille Bruno et Pascal Sellier, 1996. Dislocation en ordre paradoxal, momification et décomposition: observations et hypothèses, Bulletins et 
mémoires de la Société d'Anthropologie de Paris 8 (3-4), pp. 313-327.

OZANNE-RIVIERRE Françoise, 1997. Systèmes d'orientation: quelques exemples austronésiens, in $\mathrm{C}$. Fuchs et R. Stéphane (éds), Diversité des langues et représentations cognitives, Gap, Ophrys.

Rechtman Robert B., 1992. The evolution of sociopolitical complexity in the Fiji Islands, $\mathrm{PhD}$ dissertation, University of California, Los Angeles.

RoBineAu Claude, 2007. In memoriam José Garanger, Journal de la Société des Océanistes 125 (2), pp. 341346.

SAND Christophe and Frédérique VALENTIN, 1991. First results of the excavation of the burial mound of Petania, Uvea, Western Polynesia, Indo-Pacific Prehistory Association Bulletin 11 (2), pp. 236-246.

SAND Christophe, Frédérique Valentin et Daniel FRIMIGACCI, 2006. Sépultures à caveau en Polynésie occidentale : tradition orale et archéologie, Journal de la Société des Océanistes 122-123, pp. 13-25.

Schütz A., 1977. The diaries and correspondences of David Cargill, 1832-1843, Canberra, Australian National University Press, Pacific History Series 10.

ShutLer Mary E., Richard SHUTLER Jr and Stuart BEDFORD, 2002. Further detail on the archaeological explorations in the Southern New Hebrides, 1963-1964, in S. Bedford, C. Sand and D. Burley (eds), Fifty years in the field: Essays in honour and celebration of Richard Shutler Jr's archaeological career, Auckland, New Zealand Archaeological Association Monograph 25, pp. 189-206.

SPeISER Félix, 1996. Ethnology of Vanuatu. An early twentieth century study, Bathurst, Crawford House Press.

Spriggs Matthew, 1997. The island Melanesians, Oxford, Blackwell.

Spriggs Matthew, Fiona Petchey, Foss Leach, Stuart BEDFORD, Hallie BuCKLEY and Frédérique VALENTIN (en preparation). Direct Dating of
Lapita Skeletons from the Teouma Cemetery Site.

Testart Alain, 2004. Les morts d'accompagnements. La servitude volontaire I, Paris, Errance.

Thomas Louis-Vincent, 1980. Le cadavre, Bruxelles, Éditions Complexe.

Valentin Frédérique, Stuart Bedford, Hallie BucKLEY and Matthew SPRIGGS (en preparation). Lapita burial practices: evidence for complex body and bone treatment at the Teouma cemetery, Vanuatu, Southwest Pacific.

VALENTIN Frédérique, Isabelle Le GoFf et Christophe SAND, 2007. Le rang social des défunts inhumés au $19^{\mathrm{e}}$ siècle dans le tertre de Korotuku (Cikobia-i-Ra, Fidji), in L. Baray, P. Brun et A. Testart (eds), Pratiques funéraires et sociétés. Nouvelles approches en archéologie et en anthropologie sociale, Éditions universitaires de Dijon, coll. Art, Archéologie et Patrimoine, pp. 391-407.

VALENTIN Frédérique et Christophe SAND, 2000. Archéologie des morts. Études anthropologiques de squelettes préhistoriques de Nouvelle-Calédonie, Nouméa, Les Cahiers de l'Archéologie en Nouvelle-Calédonie 11.

VAlentin Frédérique, Richard ShIng et Matthew Spriggs, 2005. Des restes humains datés du début de la période de Mangaasi (2 400-1 800 BP) découverts à Mangaliliu (Efate, Vanuatu), Comptes Rendus Palevol 4 (5), pp. 420-427.

Ward Graeme and Philip Houghton, 1988. The Mele burials: salvage excavation and skeletal analysis, manuscript non publié.

Ward Graeme and Philip Houghton, 1991. The Mele burials (Vanuatu): salvage excavations and biological relationships, Indo-Pacific Prehistory Association Bulletin 11 (2), pp. 229-235.

Williams Thomas, 1858 (reprint 1982). Fiji and the Fijians, the islands and their inhabitants, Suva, Fiji Museum. 